\title{
Algorithm-based approach to hypervascular pancreatic lesions
}

\author{
Kheng Song Leow ${ }^{1}, M D, F R C R$, Julian Sau Lian Chieng ${ }^{1}, M D, F R C R$, Hsien Min Low ${ }^{2}$, MBBS, FRCR, \\ Kian Soon $\underline{L i m}^{3}$, FRCR, FRANZCR, Jin Wei Kwek ${ }^{4}$, MBBS, FRCR, Tze Chwan $\underline{\operatorname{Lim}}^{1}$, MBBS, FRCR, Cher Heng $\underline{\operatorname{Tan}^{2}, 5}$, MBBS, FRCR
}

\section{INTRODUCTION}

Incidental pancreatic lesions are increasingly being detected due to the increasing use of cross-sectional imaging such as computed tomography $(\mathrm{CT})$ and magnetic resonance (MR) imaging. These pancreatic lesions can be broadly categorised into hypervascular and hypovascular lesions by comparing the degree of lesion enhancement to the background pancreatic parenchymal enhancement. For practicality, we define pancreatic lesions as hypervascular when they show enhancement greater or equal to that of the pancreatic parenchyma on either CT or MR imaging vis-a-vis hypovascular lesions, which characteristically enhance less than the background pancreatic parenchyma. Hypovascular lesions have been widely described, including ductal adenocarcinoma and chronic focal pancreatitis. ${ }^{(1)} \mathrm{A}$ few prior published reviews related to hypervascular pancreatic masses have focused on the differential entities or their malignant potential. ${ }^{(2,3)}$ To the best of our knowledge, there is a lack of an algorithm-based approach that enables general radiologists to prospectively differentiate the various hypervascular pancreatic lesions, as presented in this pictorial essay. We further discuss the pertinent radiologicpathologic features of each differential diagnosis and illustrate them using individual case examples.

\section{ALGORITHM-BASED APPROACH}

Our proposed algorithm (Fig. 1) is based on the principle of 'first do no harm'. Two conditions can potentially be harmful to the patients if the correct diagnosis is not made: (a) splenic artery aneurysm or pseudoaneurysm and (b) intrapancreatic splenule. The former diagnosis is crucial so as to avoid inadvertent biopsy or fine needle aspiration, which can cause devastating bleeding. The latter is a benign, 'do not touch' lesion. ${ }^{(3)}$ Making the correct diagnosis can prevent unnecessary surgery or invasive procedures. Thus, we believe that these two conditions should be considered first and excluded. ${ }^{(3)}$

Stepwise, downwards in the algorithm, the diagnoses of pancreatic neuroendocrine tumour ( $\mathrm{pNET}$ ) and hypervascular metastasis are considered, as they are the more common conditions presenting as hypervascular lesions in the pancreas. These are followed by less common differential diagnoses such as solid-appearing serous cystic neoplasm (SCN), solid pseudopapillary epithelial neoplasm (SPEN) and acinar cell carcinoma (ACC). ACC should be the last to be considered, as it is the least common and also lacks the associated clinical characteristics; its diagnosis is often made retrospectively by histology. ${ }^{(4)}$

\section{SPLENIC ARTERY ANEURYSM OR PSEUDOANEURYSM}

Splenic artery aneurysm represents the third most common site of intra-abdominal aneurysms, following abdominal aorta and iliac arteries. A true aneurysm involves all the three walls (intima, media and adventitia) of the splenic artery, while a pseudoaneurysm usually only involves one or more walls of the splenic artery. Splenic artery aneurysms usually measure less than $3 \mathrm{~cm}$ (range 2-9 cm).

The exact pathophysiology is not known, but it is believed to be associated with systemic hypertension, portal hypertension, cirrhosis, liver transplant and pregnancy. ${ }^{(5)}$ Patients with this condition are often asymptomatic, and the lesion is usually detected incidentally. The risk of rupture is reported to be around $2 \%-3 \%$, ${ }^{(6)}$ with the rate of rupture increasing if other risk factors are present, such as size of more than $2 \mathrm{~cm}$, portal hypertension, pregnancy and being a liver transplant recipient. ${ }^{(5)}$ Management of a splenic artery aneurysm is beyond the scope of this pictorial essay. In general, for pregnant women with incidental splenic artery aneurysm, the decision for expected management, embolisation or surgery requires a balance between the risks and benefits of the treatment options, such as gestational age at time of diagnosis, size of aneurysm and the presence of symptoms. ${ }^{(7)}$ In pre-liver transplant patients (i.e. patients on the transplant waiting list), treatment of an asymptomatic aneurysm is usually postponed until the time of transplant, because the aneurysm is often small in size $(<3 \mathrm{~cm})$ and there is fear of adhesion when the splenic artery is needed for vessel anastomosis in the surgery. In post-transplant recipients, the therapeutic procedures are often complex. ${ }^{(8)}$

Pseudoaneurysms of the splenic artery usually measure more than $3 \mathrm{~cm}$, with a mean size of about $4.8 \mathrm{~cm}$ (range $0.3-17.0 \mathrm{~cm}) .{ }^{(9)}$ They are often iatrogenic and associated with trauma, pancreatitis and, rarely, peptic ulcer disease causing secondary splenic artery injury. ${ }^{(5)}$ Patients can present with 


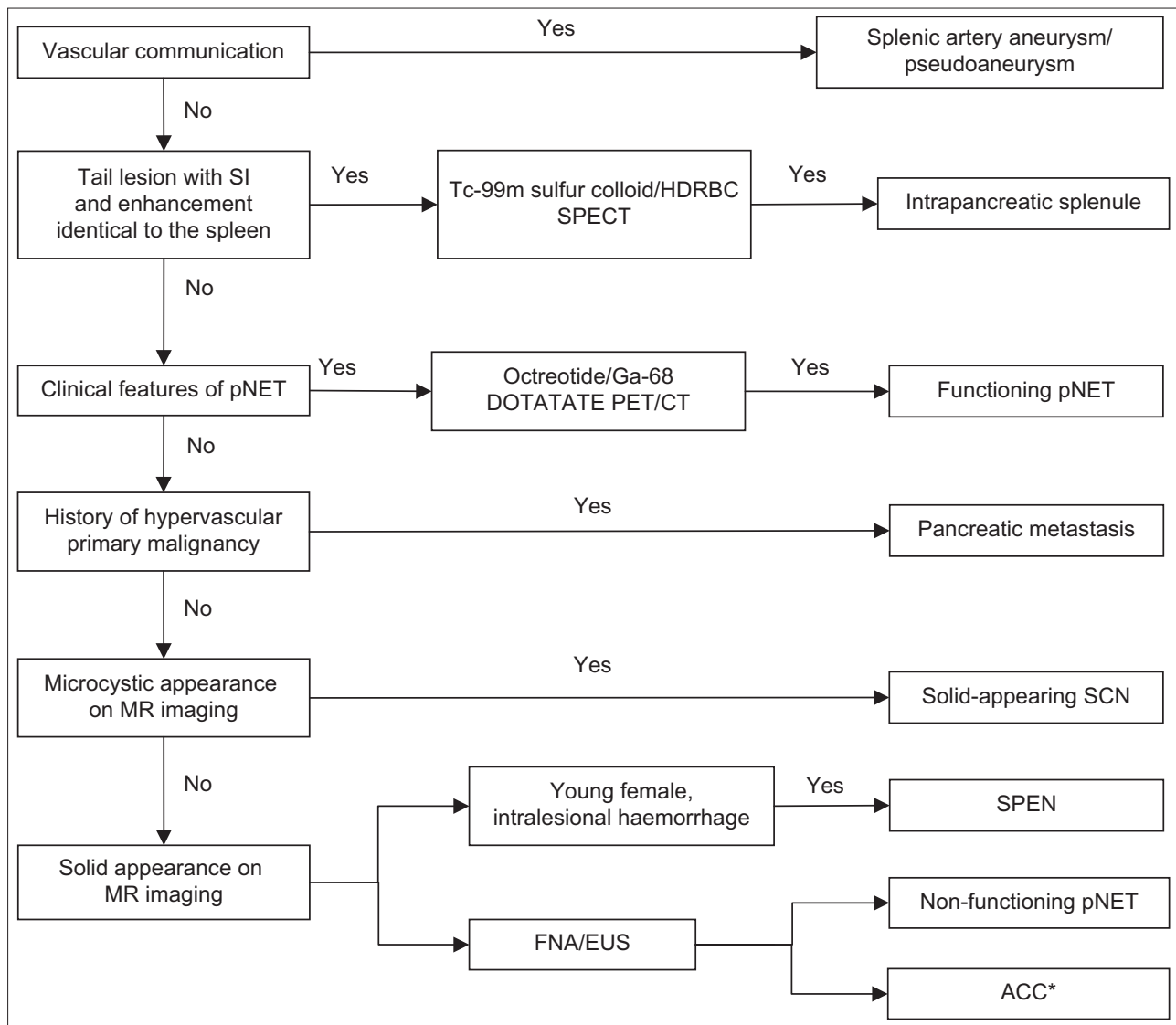

Fig. 1 Flowchart shows an algorithm-based approach to hypervascular pancreatic lesions. *Acinar cell carcinoma (ACC) is classically described as a hypovascular lesion. Based on our experience, not all ACCs demonstrate this finding; some show similar enhancement to the pancreatic parenchyma (when the central necrosis is excluded). Other reasons are discussed in the text. CT: computed tomography; EUS: endoscopic ultrasonography; FNA: fine needle aspiration; Ga-68 DOTATATE PET/CT: gallium-68 positron emission tomography/computed tomography; HDRBC: heat-damaged red blood cell; MR: magnetic resonance; pNET: pancreatic neuroendocrine tumour; SCN: serous cystic neoplasm; SI: signal intensity; SPECT: single-photon emission computed tomography; SPEN: solid pseudopapillary neoplasm; Tc-99m: technetium-99m

abdominal pain $(29.5 \%)$, haematochezia or melena $(26.2 \%)$, and haematemesis $(14.8 \%) .{ }^{(5)}$ The risk of rupture of a pseudoaneurysm is higher $(37 \%)$, with a mortality rate of about $90 \%$; thus, making a correct diagnosis and prompt treatment are important. ${ }^{(5)}$

On imaging, the diagnosis of a splenic artery aneurysm or pseudoaneurysm is often unequivocal. The lesion shows communication with the splenic artery and has a similar enhancement to that of the aorta (Fig. 2). Multiphasic imaging and multiplanar reconstruction are useful to confirm the vascular nature of the lesion (with demonstration of direct communication with the vessel) and show that the lesion does not arise from the other surrounding structures such as the stomach or left adrenal gland. Sometimes, peripheral calcification (due to atherosclerotic changes) and mural thrombus may be appreciated. ${ }^{(5)}$ In pseudoaneurysms, perilesional and peripancreatic fat stranding may be observed. ${ }^{(3)}$ The differences between a splenic artery aneurysm and pseudoaneurysm are briefly summarised in Table I.

\section{INTRAPANCREATIC SPLENULE}

Accessory spleen is a relatively common congenital condition that is often discovered incidentally on imaging performed for other causes. Previous autopsy reports suggest that it occurs in approximately $17 \%$ of the population. ${ }^{(10)}$ When an accessory spleen is found in the pancreas, the condition is called intrapancreatic splenule, which makes up approximately one sixth of all cases of accessory spleens. ${ }^{(11)}$ Familiarity with this condition is essential to avoid misdiagnosing it as a malignancy, such as neuroendocrine tumour or hypervascular metastasis, and prevent unnecessary investigations.

Intrapancreatic splenule commonly presents as a small, welldefined hypervascular lesion in the tail of the pancreas with a size of approximately $1-3 \mathrm{~cm} .{ }^{(11)}$ As it comprises the same splenic tissues as the parent spleen, it is not surprising to observe the same MR signal intensity, CT attenuation and pattern of enhancement as the parent spleen. In the arterial phase (on either CT or MR imaging), the lesion demonstrates a characteristic serpiginous appearance, similar to the parent spleen. When this feature is seen, it increases the likelihood of the diagnosis. ${ }^{(1)}$ When CT is not diagnostic, MR imaging can be performed, as it offers superior soft tissue characterisation that aids in making a more specific diagnosis (Fig. 3).

If the diagnosis remains equivocal, radionuclide studies such as technetium-99m heat-damaged red blood cell (Tc-99m HDRBC) or Tc-99m sulfur colloid scintigraphy are extremely useful, as the splenic tissue takes up and accumulates the radiotracers. Between the two nuclear medicine studies, we prefer Tc-99m HDRBC because it offers higher specificity with $90 \%$ of HDRBC uptake in the splenic tissue. ${ }^{(3)}$ The downside of 

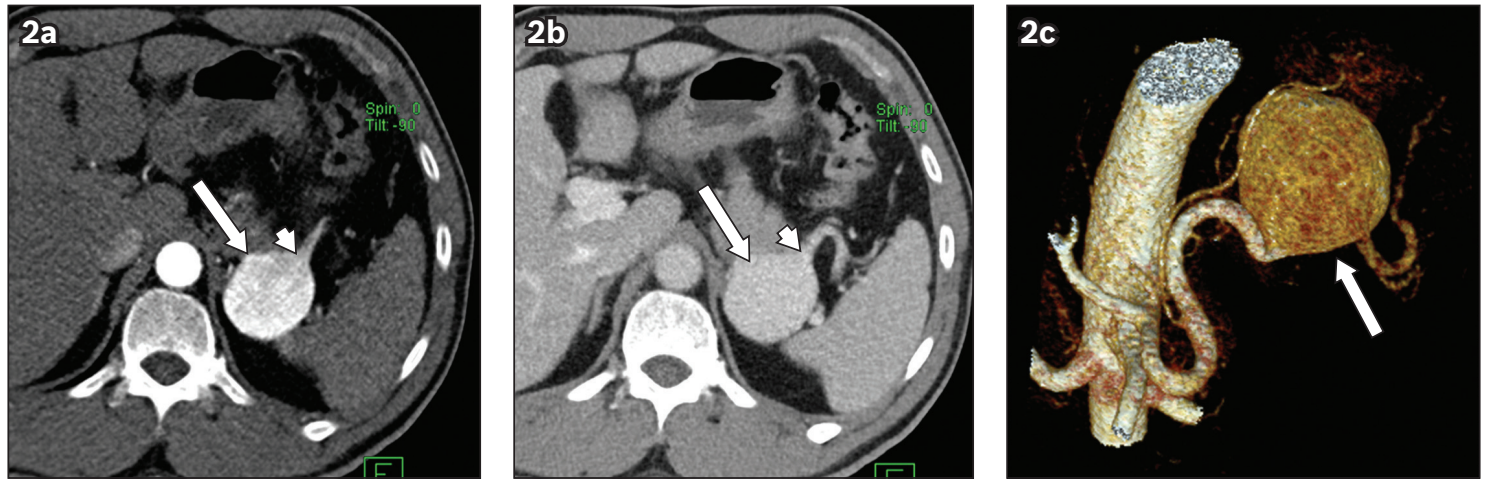

Fig. 2 Axial contrast-enhanced CT images in (a) arterial and (b) portal venous phases show a homogeneously enhancing ovoid lesion at the tail of the pancreas (arrows) with communication with the distal splenic artery (arrowheads), consistent with a splenic artery aneurysm. (c) Three-dimensional image clearly shows the communication to the splenic artery with the aneurysm (arrow).

Table I. Differences between a splenic artery aneurysm and pseudoaneurysm.

\begin{tabular}{|c|c|c|}
\hline Variable & $\begin{array}{l}\text { Splenic artery } \\
\text { aneurysm }\end{array}$ & Pseudoaneurysm \\
\hline Pathophysiology & Unknown & $\begin{array}{l}\text { - Trauma } \\
\text { - Pancreatitis } \\
\text { - latrogenic } \\
\text { - Peptic ulcer } \\
\text { disease }\end{array}$ \\
\hline Clinical & $\begin{array}{l}\text { Asymptomatic } \\
\text { (unless ruptured) }\end{array}$ & $\begin{array}{l}\text { - Abdominal pain } \\
\text { - Bleeding }\end{array}$ \\
\hline CT/MR imaging & $\begin{array}{l}\text { - Communicating } \\
\text { with splenic artery } \\
\text { - Enhancement } \\
\text { similar to aorta } \\
\text { - Peripheral } \\
\text { calcification }\end{array}$ & $\begin{array}{l}\text { - Same } \\
\text { - Background } \\
\text { acute/chronic } \\
\text { pancreatitis }\end{array}$ \\
\hline $\begin{array}{l}\text { Indication for } \\
\text { treatment }\end{array}$ & $\begin{array}{l}\text { - Symptomatic } \\
\text { - Aneurysm > } 2 \mathrm{~cm} \\
\text { - Portal hypertension } \\
\text { - Pregnant woman } \\
\text { - Recipient of liver } \\
\text { transplant }\end{array}$ & $\begin{array}{l}\text { - Earliest possible } \\
\text { intervention (high } \\
\text { risk of rupture) }\end{array}$ \\
\hline
\end{tabular}

$\mathrm{CT}$ : computed tomography; MR: magnetic resonance

Tc-99m HDRBC is the need to handle blood products and the longer scanning time. ${ }^{(3)}$

\section{PANCREATIC NEUROENDOCRINE TUMOUR}

PNETs arise from the endocrine cells of the pancreas ${ }^{(3)}$ and commonly present in individuals aged $50-70$ years. ${ }^{(12)}$ pNETs are categorised clinically as functional and non-functional, depending on the presence or absence of symptoms associated with excessive secretion of hormones (e.g. insulin, glucagon, gastrin, vasoactive intestinal peptide and somatostatin). Most of the pNETs detected nowadays are non-functional $(\sim 60 \%-$ $80 \%$ ) due to the increasing use of cross-sectional imaging. Non-functional PNETs have variable malignant potential, ranging from slow growing and indolent to being aggressively metastasising lesions. ${ }^{(3)}$ pNETs can occur sporadically or arise in association with genetic syndromes such as von Hippel-Lindau disease, multiple endocrine syndrome Type 1 , tuberous sclerosis and neurofibromatosis. ${ }^{(3)}$

On contrast-enhanced CT or MR imaging, pNETs commonly present as a well-circumscribed hypervascular lesion in the pancreas with a different degree of enhancement compared to the spleen, thus excluding the diagnosis of intrapancreatic splenule (Fig. 4). Larger pNETs tend to show a central area of hypoenhancement. The pancreatic duct is usually normal in calibre, unless the lesion is large and causes secondary extrinsic compression of the duct. ${ }^{(12)}$ If MR imaging is performed, its characteristic high T2-weighted signal intensity, which is close to the fluid signal intensity and similar to that of the normal spleen, can be depicted. ${ }^{(12)}$

When the diagnosis is equivocal, radioisotope studies such as Indium-111 octreotide scintigraphy or the currently preferred gallium-68 (Ga-68) DOTATATE positron emission tomography (PET/CT), are useful. Ga-68 DOTATATE PET/CT has a higher diagnostic accuracy with a sensitivity of $93 \%$ and specificity of $96 \% .{ }^{(13)}$ As making the diagnosis based on imaging alone can sometimes be difficult, tissue sampling may be required in some cases. ${ }^{(3)}$

Although several non-benign features have been described in the literature (e.g. hypovascularity, cystic morphology, presence of calcification, pancreatic ductal dilatation, adenopathy and size $>3 \mathrm{~cm}$ ), the aggressive behaviour of pNETs often requires histopathology to assess for mitotic activity and the percentage of nuclear antigen Ki-67. ${ }^{(12)}$ Nevertheless, if locoregional invasion and liver or nodal metastases are seen on imaging, the diagnosis of a malignant pNET should be raised. ${ }^{(12)}$

\section{HYPERVASCULAR METASTASES}

Metastasis to the pancreas is rare, consisting of $2 \%-5 \%$ of all pancreatic malignancies. ${ }^{(14)}$ The most common primary malignancy that metastasises to the pancreas is renal cell carcinoma (RCC), followed by lung, breast and colorectal malignancies, as well as melanoma. ${ }^{(3)}$ Delayed pancreatic metastasis occurring more than ten years after the initial diagnosis of RCC has been reported and can happen in more than $10 \%$ of patients. About half of the patients with pancreatic metastases are asymptomatic, while the other half may present with abdominal pain, obstructive jaundice or pancreatitis. ${ }^{(14)}$ 

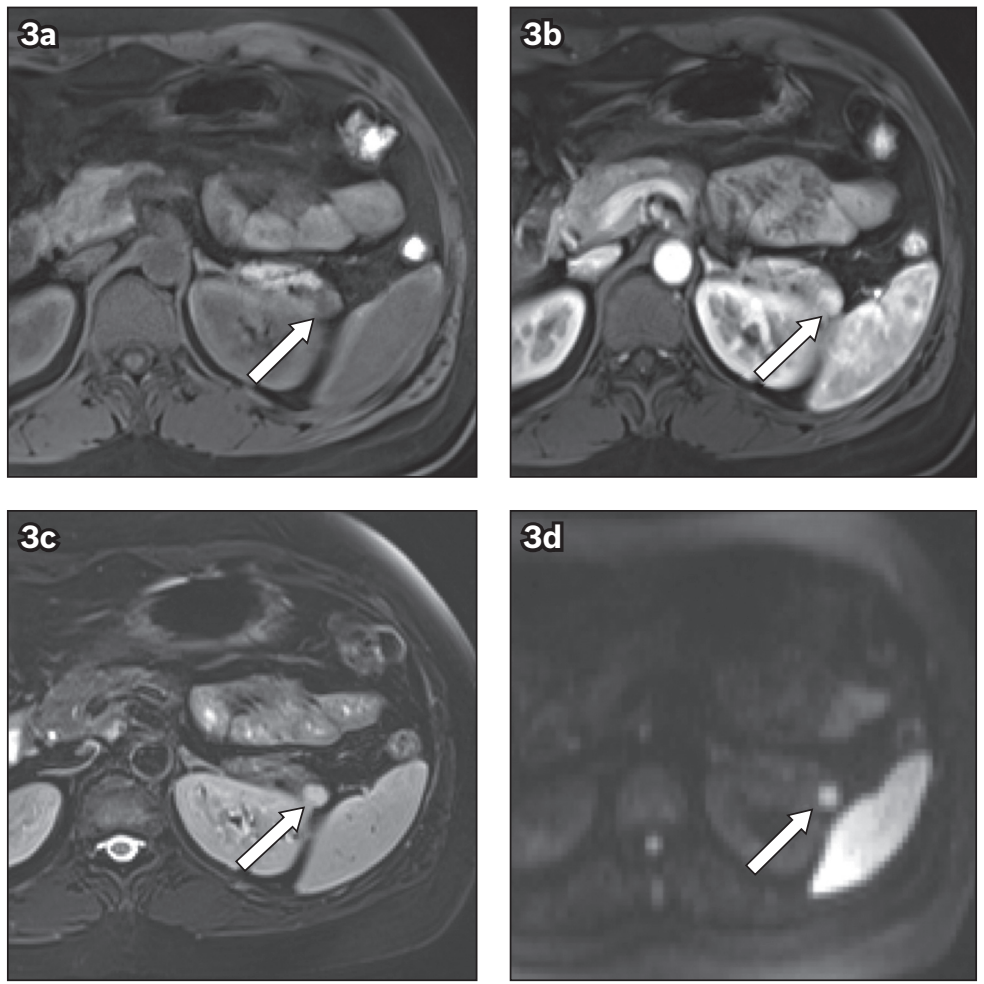

Fig. 3 Incidental hypervascular lesion found at the tail of the pancreas (arrows) shows signal intensities and degree of contrast enhancement similar to that of the adjacent spleen on the axial sections of (a) fat-suppressed T1-weighted pre-contrast, (b) arterial phase, (c) portal venous phase and (d) diffusion-weighted images. The findings are consistent with those of an intrapancreatic splenule.
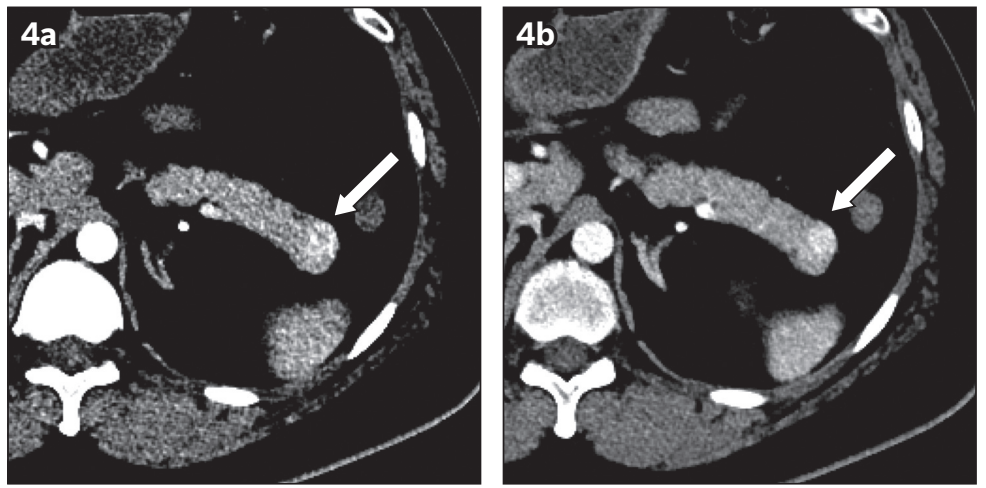

Fig. 4 A 38-year-old man presented with hypoglycaemia. Axial contrast-enhanced CT images in (a) arterial and (b) portal venous phases show a hyperenhancing lesion at the tail of the pancreas with a degree of enhancement greater than that of the adjacent spleen (arrows). The lesion was histologically confirmed to be a well-differentiated neuroendocrine tumour (insulinoma).

Imaging features of hypervascular pancreatic metastases can closely mimic those of primary pNETs, presenting as a welldefined hypervascular lesion without main pancreatic ductal dilatation. ${ }^{(14)}$ However, metastases to the pancreas often manifest with multiplicity and patients often have a previous history of hypervascular primary malignancies or evidence of nephrectomy, which suggests a prior RCC (Fig. 5). ${ }^{(3)}$ Thus, careful evaluation of the organs (e.g. kidneys, lungs and breasts) for evidence of primary malignancy is extremely important when multiple hypervascular pancreatic lesions are seen.

\section{SOLID-APPEARING SEROUS CYSTIC NEOPLASM}

As the name implies, SCN of the pancreas usually presents as a cystic lesion in about $80 \%$ of cases. ${ }^{(15)}$ It is referred to as the 'grandmother' lesion, classically occurring in women above 60 years of age and typically located in the head of the pancreas, as compared to the mucinous cystic neoplasm, which is more commonly seen in the body and tail of the pancreas in women aged $40-50$ years. ${ }^{(15)}$

In a minority (about 20\%), SCNs can present as solidappearing lesions due to their closely apposed enhancing septae and walls. ${ }^{(3)}$ In this situation, T2-weighted MR images are extremely helpful in establishing the microcystic nature of the pseudo-solid lesion. SCNs tend to have a lobulated margin along with microcystic morphology, giving rise to their honeycomb appearance. Sometimes, a central scar or calcification may be seen, adding to diagnostic confidence (Fig. 6). ${ }^{(15)}$ Very few serous cystadenocarcinomas have been reported to turn malignant. ${ }^{(15)}$ 

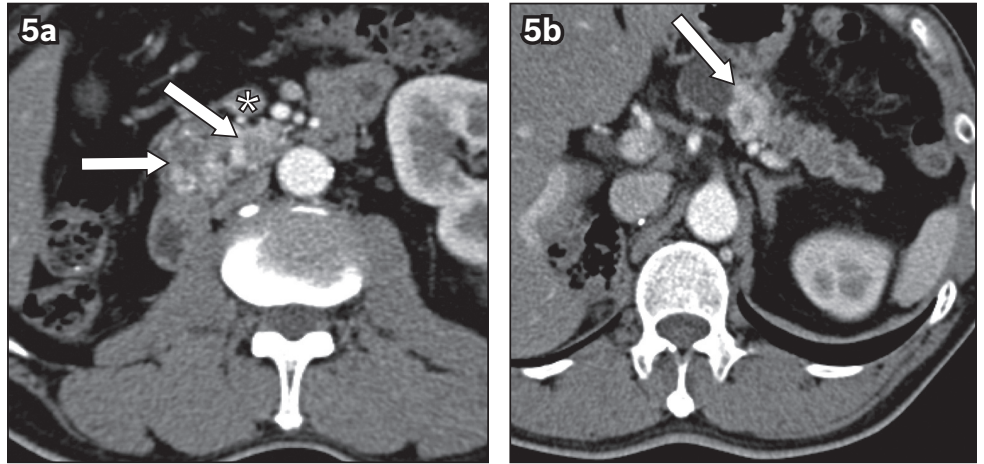

Fig. 5 Hypervascular metastases from renal cell carcinoma. (a \& b) Axial contrast-enhanced CT images of a patient with previous right nephrectomy for renal cell carcinoma show multiple hyper-enhancing lesions in the pancreas (arrows), some with necrotic centres, suspicious for hypervascular pancreatic metastases. The superior mesenteric vein (asterisk) is seen. Note the absence of the right kidney.
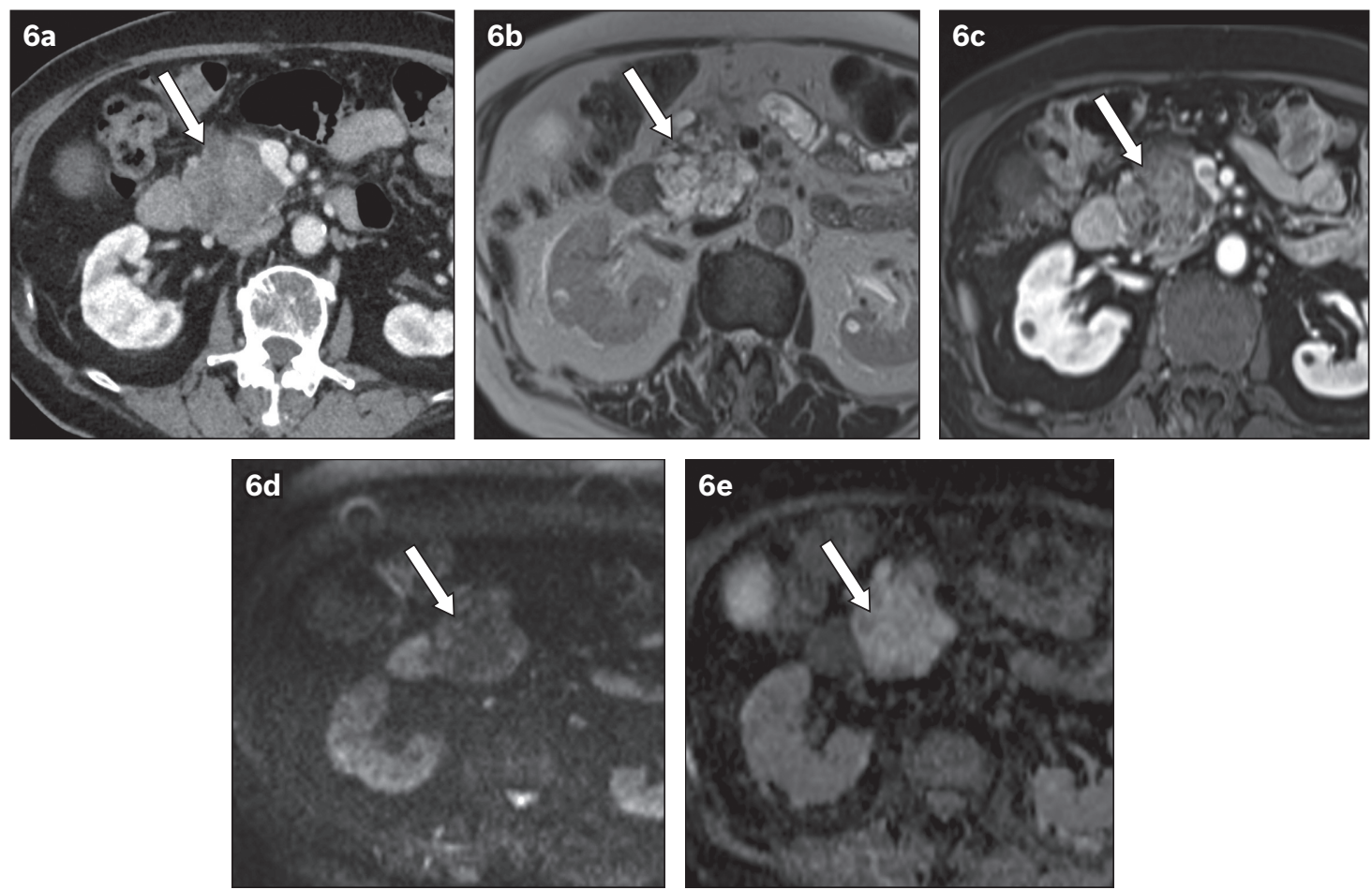

Fig. 6 (a) Axial contrast-enhanced CT image shows a solid-appearing heterogeneous mass in the head of the pancreas (arrow). (b) T2-W image shows a lobulated microcystic lesion with central scar (arrow). (c) T1-W post-contrast image shows enhancement of the septae and central component (arrow). (d) Diffusion-weighted and (e) apparent diffusion coefficient images show T2 shine-through without restricted diffusion (arrows). The constellation of findings suggests serous cystic neoplasm of the pancreas.

\section{SOLID PSEUDOPAPILLARY EPITHELIAL NEOPLASM}

SPEN occurs almost exclusively in young women aged below 40 years. ${ }^{(16)}$ Patients are often asymptomatic, but if the mass is large, some may present with abdominal distension or vague abdominal pain. SPEN is recognised as an indolent tumour with potential malignant behaviour (harbouring up to approximately $10 \%-20 \%$ malignant potential). ${ }^{(16)}$ In our experience, middle-aged men can sometimes present with this pathology.

Typically, SPEN presents as a well-defined solid mass in the tail of the pancreas (Fig. 7). The mass can be heterogeneous due to internal haemorrhage and necrosis. If MR imaging is performed, a T1-weighted sequence is useful to confirm the presence of internal blood products (with high T1 signals representing subacute methaemoglobin), while T2-weighted imaging is useful to demonstrate a thick T2 hypointense capsule composed of fibrous capsule and compressed pancreatic tissue. ${ }^{(17)}$ When capsular/parenchymal invasion, perineural/vascular invasion or liver metastasis is seen, malignant differentiation should be considered. In such cases, it becomes harder to make a specific diagnosis based on imaging alone, and thus tissue sampling, such as fine needle aspiration or biopsy, is recommended. ${ }^{(16)}$

\section{ACINAR CELL CARCINOMA}

ACC is a rare epithelial neoplasm of the pancreas with acinar cell differentiation. ${ }^{(4)}$ It is an aggressive tumour with high malignant risk. ${ }^{(3)}$ It typically occurs in elderly male patients (aged 50-70 years). The clinical symptoms are often non-specific, with abdominal pain being the most common presentation (about $55 \%$ ), followed by nausea/vomiting, loss of weight and loss of appetite. Uncommonly, patients with ACC can present with paraneoplastic syndromes due to hyperlipasaemia, manifesting 

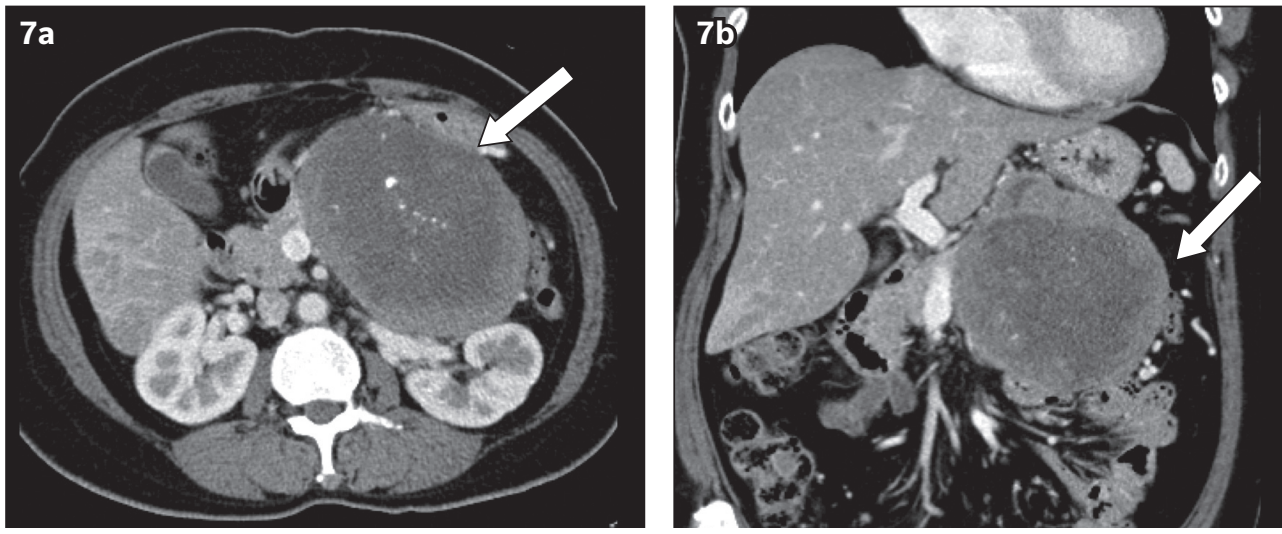

Fig. 7 (a) Axial and (b) coronal contrast-enhanced CT images of the abdomen in a 35-year-old woman show a large heterogeneously and peripherally enhancing mass (arrows) at the distal pancreas with a well-defined margin, necrotic centre and internal coarse calcifications. It was histologically diagnosed to be a solid pseudopapillary tumour of the pancreas.
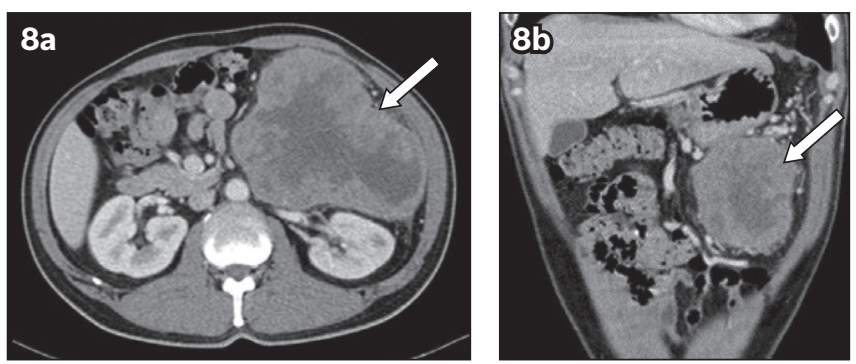

Fig. 8 (a) Axial and (b) coronal CT images of an elderly man show a large exophytic heterogeneously and peripherally enhancing mass at the tail of the pancreas (arrow). The mass has a necrotic centre, and is distinct from and exerts mass effect on the surrounding structures (e.g. left kidney, stomach and bowel loops). It was histologically diagnosed to be acinar cell carcinoma of the pancreas.

as painful and erythematous subcutaneous nodules and multiple lytic lesions involving the peripheral joints (e.g. wrists, hands, ankle, feet and knees), and mimicking metastases. ${ }^{(4)}$

Although ACC is typically described in the literature as a large $(>4 \mathrm{~cm}$ ) exophytic mass in the pancreas with heterogeneous enhancement and a well-defined margin (Fig. 8), ${ }^{(4)}$ based on our experience, it is often not easy to make an accurate prospective diagnosis based on imaging alone. ACC has been classically described as hypovascular. We believe it may be attributed to central necrosis. We have included this differential diagnosis in the algorithm for three reasons. First, not all of the ACCs that we have encountered are hypovascular - some demonstrate enhancement similar to that of the pancreatic parenchyma, excluding the central necrotic component. Secondly, it is an aggressive tumour with malignant potential; therefore, we do not want to misdiagnose this lesion in the first encounter. Lastly, since the morphological features are often non-specific and can be confused with the aforementioned conditions, histological correlation will no doubt be required.

\section{CONCLUSION}

Hypervascular pancreatic lesions have a range of differential diagnoses from benign to malignant conditions. We have described, with specific examples, an algorithm-based approach that will enable radiologists to make a sensible and appropriate prospective diagnosis in daily clinical practice.

\section{REFERENCES}

1. Kim JH, Lee JM, Park JH, et al. Solid pancreatic lesions: characterization by using timing bolus dynamic contrast-enhanced MR imaging assessment--a preliminary study. Radiology 2013; 266:185-96.

2. Bhosale PR, Menias CO, Balachandran A, et al. Vascular pancreatic lesions: spectrum of imaging findings of malignant masses and mimics with pathologic correlation. Abdom Imaging 2013; 38:802-17

3. Shankar PR, Wasnik AP, Al-Hawary MM, Francis IR, Kaza RK. Hypervascular pancreatic "lesions": a pattern-based approach to differentiation. Abdom Radiol (NY) 2018; 43:1013-28

4. Tatli S, Mortele KJ, Levy AD, et al. CT and MRI features of pure acinar cell carcinoma of the pancreas in adults. AJR Am J Roentgenol 2005; 184:511-9.

5. Agrawal GA, Johnson PT, Fishman EK. Splenic artery aneurysms and pseudoaneurysms: clinical distinctions and CT appearances. AJR Am J Roentgenol 2007; 188:992-9.

6. Trastek VF, Pairolero PC, Bernatz PE. Splenic artery aneurysms. World J Surg 1985; 9:378-83.

7. Wiener $Y$, Tomashev R, Neeman $O$, et al. Splenic artery aneurysms during pregnancy: an obstetric nightmare. Eur J Obstet Gynecol Reprod Biol 2019; 237:121-5.

8. Maggi U, Dondossola D, Consonni D, et al. Visceral artery aneurysms in liver transplant candidates and in patients after liver transplantation. PLoS One 2011; 6:e29544.

9. Tessier DJ, Stone WM, Fowl RJ, et al. Clinical features and management of splenic artery pseudoaneurysm: case series and cumulative review of literature. J Vasc Surg 2003; 38:969-74.

10. Halpert B, Gyorkey F. Lesions observed in accessory spleens of 311 patients. Am J Clin Pathol 1959; 32:165-8.

11. Spencer LA, Spizarny DL, Williams TR. Imaging features of intrapancreatic accessory spleen. Br J Radiol 2010; 83:668-73.

12. Gallotti A, Johnston RP, Bonaffini PA, et al. Incidental neuroendocrine tumors of the pancreas: MDCT findings and features of malignancy. AJR Am J Roentgenol $2013 ; 200: 355-62$.

13. Geijer H, Breimer LH. Somatostatin receptor PET/CT in neuroendocrine tumours: update on systematic review and meta-analysis. Eur J Nucl Med Mol Imaging 2013; 40:1770-80.

14. Vincenzi M, Pasquotti G, Polverosi R, Pasquali C, Pomerri F. Imaging of pancreatic metastases from renal cell carcinoma. Cancer Imaging 2014; 14:5

15. H'ng MWC, Kwek JW, Liao KH, Vu CK. Cystic pancreatic lesions: a pictorial review and management approach. Singapore Med J 2010; 51:668-75.

16. De Robertis R, Marchegiani G, Catania M, et al. Solid pseudopapillary neoplasms of the pancreas: clinicopathologic and radiologic features according to size. AJR Am J Roentgenol 2019; 213:1073-80.

17. Mortele BP, Mortele KJ, Tuncali K, et al. Solid and papillary epithelial neoplasm of the pancreas: MR imaging findings. JBR-BTR 2002; 85:297-9. 


\section{SINGAPORE MEDICAL COUNCIL CATEGORY 3B CME PROGRAMME} (Code SMJ 202103B)

1. Most pancreatic neuroendocrine tumours (pNETs) are hypervascular in nature.

2. In this pictorial essay, pancreatic lesions are defined as hypervascular when they demonstrate greater or equal enhancement to the background pancreatic parenchyma.

3. Splenic artery pseudoaneurysms involve all the three walls of the artery.

4. Splenic artery aneurysms are usually smaller than $3 \mathrm{~cm}$.

5. Splenic artery aneurysms and pseudoaneurysms typically demonstrate similar enhancement to the aorta and communicate with the splenic arteries.

6. An intrapancreatic splenule commonly presents as a well-defined hypervascular lesion with enhancement patterns and magnetic resonance (MR) signal intensities similar to those of the spleen.

7. Technetium-99m heat-damaged red blood cell or sulfur colloid studies are useless in the diagnosis of intrapancreatic splenule.

8. All pNETs are benign.

9. Due to the increasing use of cross-sectional imaging such as computed tomography (CT), most of the pNETs that are detected nowadays are non-functional.

10. pNETs can be associated with genetic syndromes such as von Hippel-Lindau and multiple endocrine syndrome Type 1.

11. In equivocal cases with strong clinical suspicion of pNETs, gallium-68 DOTATATE positron emission tomography/CT study is useful for further evaluation.

12. When multiple hypervascular lesions are seen in the pancreas, the first and most sensible diagnosis is PNET of the pancreas.

13. The most common primary malignancy that metastasises to the pancreas is renal cell carcinoma.

14. Serous cystic neoplasms always present with an obvious cystic mass in the head of the pancreas on CT.

15. When an elderly woman presents with a cystic pancreatic head lesion with a central scar and calcification, the most likely diagnosis is mucinous cystic neoplasm of the pancreas.

16. T1-weighted sequence is extremely useful to confirm the presence of subacute blood products when a solid pseudopapillary epithelial neoplasm (SPEN) is suspected in a pancreatic tail lesion in a young female patient.

17. When SPENs are associated with capsular/parenchymal invasion, perineural/vascular invasion or liver metastasis, malignant differentiation should be considered.

18. Acinar cell carcinoma (ACC) is a common epithelial neoplasm of the pancreas.

19. ACC is a benign neoplasm of the pancreas.

20. Although ACCs are classically described as a hypovascular lesion of the pancreas, they are included in the algorithm because (a) not all ACCs are hypovascular when the central necrotic component is excluded; (b) non-specific morphological imaging features of ACCs still require histological correlation; and (c) to avoid early dismissal of this diagnosis by inexperienced radiologists.

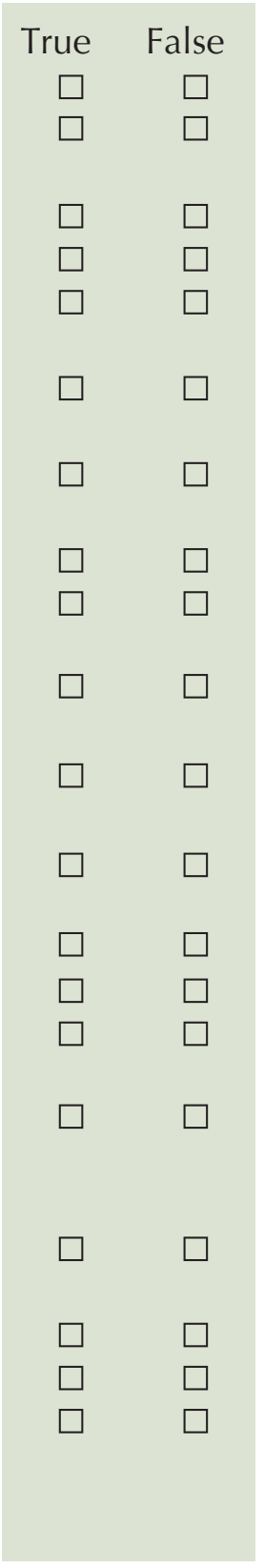

\section{Doctor's particulars:}

Name in full:

MCR no.:

Specialty:

Email:

\section{SUBMISSION INSTRUCTIONS:}

Visit the SMJ website: http://www.smj.org.sg/current-issue and select the appropriate quiz. You will be redirected to the SMA login page.

For SMA member: (1) Log in with your username and password (if you do not know your password, please click on 'Forgot your password?'). (2) Select your answers for each quiz and click 'Submit'.

For non-SMA member: (1) Create an SMJ CME account, or log in with your SMJ CME username and password (for returning users). (2) Make payment of SGD 21.40 (inclusive of $7 \%$ GST) via PayPal to access this month's quizzes. (3) Select your answers for each quiz and click 'Submit'.

\section{RESULTS:}

(1) Answers will be published online in the SMJ May 2021 issue. (2) The MCR numbers of successful candidates will be posted online at the SMJ website by 10 May 2021. (3) Passing mark is $60 \%$. No mark will be deducted for incorrect answers. (4) The SMJ editorial office will submit the list of successful candidates to the Singapore Medical Council. (5) One CME point is awarded for successful candidates. (6) SMC credits CME points according to the month of publication of the CME article (i.e. points awarded for a quiz published in the March 2021 issue will be credited for the month of March 2021, even if the deadline is in May 2021).

Deadline for submission (March 2021 SMJ 3B CME programme): 12 noon, 3 May 2021. 